\title{
Parallel Assembly of Hinged Microstructures Using Magnetic Actuation
}

\author{
Yong Yi and Chang Liu \\ Microelectronics Laboratory \\ University of Illinois at Urbana-Champaign \\ Urbana, IL 61801
}

\begin{abstract}
We have developed a new surface-micromachining process for realizing three-dimensional MEMS devices from hinged planar structures. Magnetic actuation, which is provided by Permalloy material attached to hinged plates, provides efficient and highyield out-of-plane actuation. Parallel actuation of large arrays of hinged microstructures is achieved under a globally applied external magnetic field. The magnitude of the actuation can be controlled by either the volume of the magnetic piece or the stiffness of a flexural-beam loading spring. Asynchronous displacement of hinged structures allow these devices to lock into three dimensional assemblies that remain stable after the activation magnetic field is removed.
\end{abstract}

\section{INTRODUCTION}

It has been demonstrated that three-dimensional MEMS devices can be attained through assembly of hinged microstructures [1], which are capable of rotation about their base. Potential applications of assembled 3-D devices include micro opto-electro-mechanical components and fluidic turbulators, to name a few. Existing techniques for achieving displacements include water rinse, scratch drive actuation [2], and thermal actuation [3]. However, these methods do not offer sufficient reliability and efficiency. In most developed methods using active actuation, one actuator is connected to only a single hinged plate; the efficiency is low for deploying large arrays of devices. These actuators also occupy significant chip area. In order to accomplish mass fabrication of array 3-D MEMS, a high yield and efficient process for activating and assembling hinged structures must be developed.

In this paper, we present a new process for actuation and assembly of hinged structures based on magnetic actuation. Detailed mechanisms are described in the following section. Parallel assembly using magnetic forces offers the following unique advantages: (1) high yield; (2) low chip-area requirements and high device density; (3) efficient wafer-scale assembly. A globally applied external magnetic field $\left(H_{\text {ext }}\right)$ can address a large number of devices simultaneously. A linear sweep of the magnitude of $H_{\text {ext }}$ induces the formation of three-dimensional structures, which remain stable even after $H_{e x t}$ has been removed. The actuation mechanism is relatively simple and exhibits higher efficiency and yield compared with other afore-mentioned methods.

\section{DESIGN AND THEORY}

A piece of magnetic material (Permalloy) is attached to each hinged microstructure (so called flap) by an electroplating process to provide a means of actuation (Fig. 1). Each flap is capable of rotation about its base. The electroplating step follows established hinge-fabrication processes (such as MUMPS) and has no influence on existing designs and recipes. If an external magnetic field is applied perpendicular to the substrate (Fig. 1c), the Permalloy material develops a magnetization, $M$. It subsequently interacts with $H_{\text {ext }}$ to induce a torque, $T$, on the hinged structure and causes it to deflect away from the substrate plane. The magnitude of $T$ is given by:

$$
T=M A H_{\text {exl }} l_{\text {mag }} \cos \theta
$$

where $A$ and $l_{\text {mag }}$ are the cross-sectional area and the length of the Permalloy piece, respectively, and $\theta$ represents the angle between the flap and the substrate.

In order for hinged flaps to assemble into a predetermined 3D shape, it is important to control the speed of actuation of each individual flap component, so that they exhibit different displacement under a global magnetic field. In our work, this has been realized using two methods. First, the volume and orientation of the electroplated magnetic piece determine the magnitude of the magnetic torque and, subsequently, the displacement angle, under a given $H_{\text {ext. }}$ In the second method, a cantilever-beam spring loading mechanism is developed to provide a resistive force to the otherwise free flap as it rotates out of plane. By using these two methods, individual hinged components can be lifted in a predetermined asynchronous order.

Two types of hinged structures have been designed, based on the control methods described earlier. Type I structures consist of a flap which is hinged at the base and may rotate without restriction (except for friction) about the $y$-axis (Fig. 1).

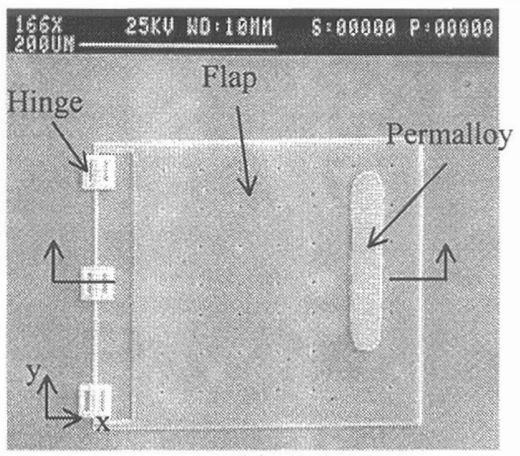

(a) (b)

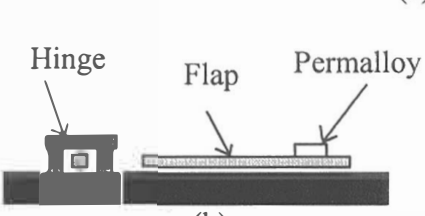

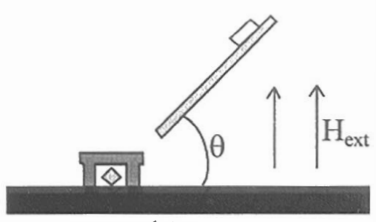

(c)
Figure 1. (a) An SEM micrograph of a Type I structure. The flap is allowed to rotate about the y-axis. (b) Schematic cross-sectional view of the structure at rest; (c) schematic cross-sectional view of the flap as $H_{\text {ext }}$ is increased. 
A Type II structure, on the other hand (Fig. 2), consists of an offset slab and a fixed cantilever-beam loading spring. When the flap is at rest $\left(\theta=0^{\circ}\right)$, the offset slab and the cantilever beam are not in contact. As the flap is rotated away from plane, the offset slab, which is wider than average hinge pins, comes into contact with the loading spring and experiences a reactive force due to the bent cantilever beam. The vertical deflection of the cantilever beam at its free end, $y_{\max }$, is related to the reactive force, $F$, using small-bending assumptions [4]:

$$
y_{\max }=\frac{F l^{3}}{3 E I}
$$

where $l$ is the effective length of the beam, $E$ is the modulus of elasticity, and $I$ is the moment of inertia of the cantilever beam. $I$ equals $w h^{3} / 12$, where $w$ is the base width and $h$ is the thickness of beam

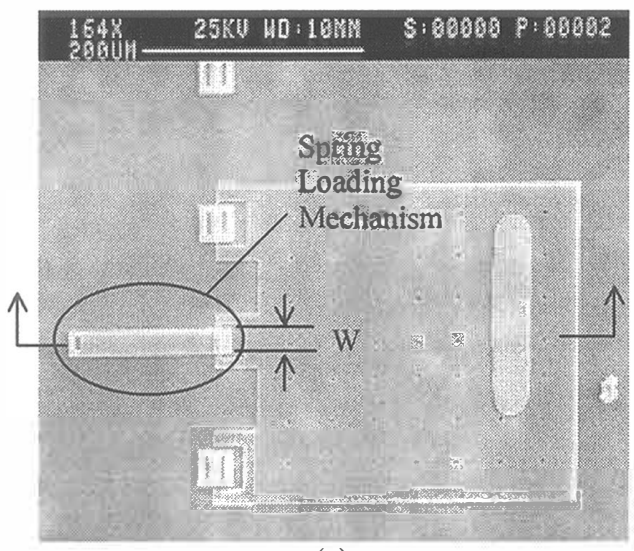

(a)

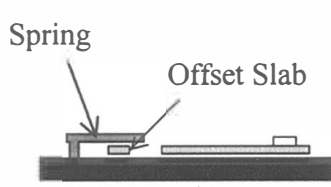

(b)

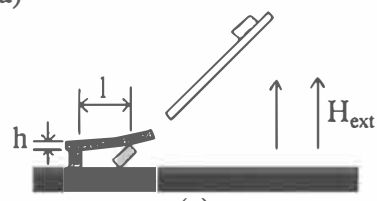

(c)
Figure 2. (a) SEM micrograph of the Type II structure; (b) schematic cross-sectional view of the structure at rest; (c) schematic cross-sectional view of the structure when $H_{\text {ext }}$ is increased.

The geometry of the cantilever beam, therefore, determines the spring constant and the magnitude of the resistance to the flap displacement. Tests were conducted for cantilever beams with lengths of 75,100 , and $125 \mu \mathrm{m}$ and widths of 15,25 , and $35 \mu \mathrm{m}$. Dimensions of the flaps for both types are $300 \times 300 \times 2 \mu \mathrm{m}^{3}$. The dimensions of the Permalloy pieces are $200 \times 40 \times 2.1 \mu^{3}$.

Assemblies of 3-D devices using Type I structures have been accomplished based on asynchronous actuation. One mechanism involves two flaps and requires a precise sequence of actuation to assemble. The second mechanism involves three flaps; the order of actuation is not critical in this assembly process. Detailed mechanisms for locking and assembly will be discussed in the results section.

\section{FABRICATION}

Hinged flaps and assemblies can be fabricated based on established surface micromachining processes such as the MCNC
MUMPS. The major steps in the process are shown in Figure 3. To form the flap, a layer of polysilicon is deposited and patterned on top of a sacrificial oxide. A second oxide layer is grown and vias are etched so that hinges can anchor to the substrate. The final step in structure fabrication is to deposit and pattern a second polysilicon layer, forming the hinge.

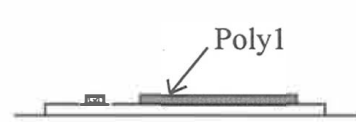

(a)

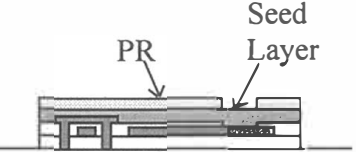

(c)

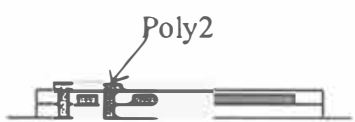

(b)

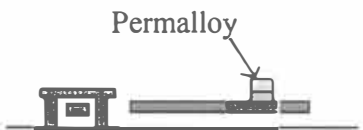

(d)
Figure 3. Major steps in the fabrication process for the hinged microstructures (not to scale). (a) Deposition of polysilicon as the structural layer of the flap; (b) deposition of polysilicon for the hinge; (c) deposit seed layers and pattern $P R$ to prepare for electroplating; (d) electroplate Permalloy and release structure.

A piece of magnetic material (Permalloy, $\mathrm{Ni}_{80} \mathrm{Fe}_{20}$ ) is then attached to the flap [5]. The first step is to expose the first polysilicon layer by selectively opening the second oxide. Layers of $\mathrm{Cr}$ and $\mathrm{Cu}$ are then deposited as the seed layer for the electroplating process. Because $\mathrm{Cu}$ does not adhere well to the substrate, an initial $200 \AA$-thick $\mathrm{Cr}$ layer is sputtered to act as an adhesion promoter between polysilicon and $\mathrm{Cu}$. A layer of $\mathrm{Cu}$ $(4000 \AA)$ is evaporated to provide adequate electrical continuity for the electroplating process. A $6.2 \mu \mathrm{m}$ layer of photoresist is patterned on the seed layer and the chip is mold electroplated to the desired thickness.

After the magnetic material has been electroplated, the PR and the exposed seed layer materials are removed. The structures are then released in $\mathrm{HF}$ ( $49 \%$ wt.) and rinsed in DI water. The patterned Permalloy is unaffected throughout this process as it is resistive to $\mathrm{HF}, \mathrm{Cu}$, and $\mathrm{Cr}$ etchants. The final step is to place the chip in isopropyl alcohol and allow the solution to dry in air. As the isopropyl alcohol evaporates, surface tensions of the liquid can force the flaps to come into contact with the substrate and cause stiction. To remedy this, a magnetic field $\left(8.1 \times 10^{4} \mathrm{~A} / \mathrm{m}\right)$ is applied to the chip so that the flaps are rotated by $90^{\circ}$ during the liquid evaporation; the magnetic field is maintained until the isopropyl alcohol is completely evaporated. This method, also used in [6], significantly increases the yield.

\section{EXPERIMENTAL SETUP}

Measurements of flap angular displacement vs. $H_{e x t}$ are obtained using a video-microscopy setup illustrated in Fig. 4. The camera is placed at an angle, $\alpha$, with respect to the wafer substrate. The estimated error for the measurements in the angular displacement is $\pm 5^{\circ}$. The tests are conducted with the structures immersed in isopropyl alcohol and placed on top of an electromagnet. The purpose of isopropyl alcohol is to minimize frictional effects at the base and potential stiction between the flap and substrate.

The tilted viewing angle and the refraction at the liquid-air interface causes the perceived displacement angle, $\beta$, to be different from the actual displacement angle, denoted $\theta$. The relationship between $\beta$ and $\theta$ can be expressed by: 


$$
\tan \theta=\frac{\tan \beta \tan \left[\cos ^{-1}\left(\frac{n_{1}}{n_{2}} \cos \alpha\right)\right]}{\sin \alpha}
$$

where $n_{1}$ and $n_{2}$ are the refractive indices for air and isopropyl alcohol, respectively.

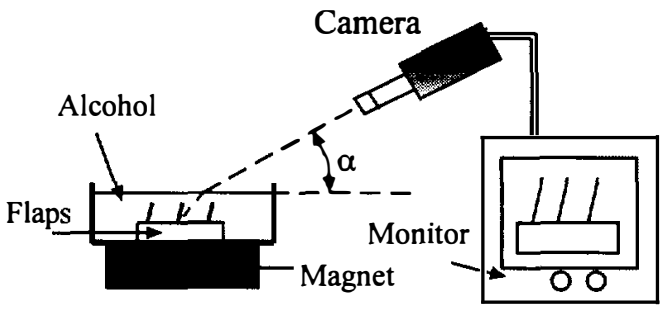

Figure 4. Experimental setup. The chip is immersed in alcohol. The CCD camera views the structures at an inclined angle $\alpha$. A magnified image of the structures is displayed on an external monitor. Angular displacement measurements are taken from the monitor.

\section{RESULTS AND DISCUSSION}

Within an external magnetic field, Type I structures behave in a binary fashion; they can assume only two stable positions, $0^{\circ}$ or $90^{\circ}$. The flaps are initially in plane, $\theta=0^{\circ}$, when no $H_{\text {ext }}$ is applied. As $H_{e x t}$ increases, the flap remains at $0^{\circ}$ until a threshold magnetic field strength $\left(H_{t}\right)$ is reached. At $H_{t}$, the flap makes a direct transition to $90^{\circ}$. Figure 5 shows the measured $H$, values for three separate Type I structures of identical geometries within ten trials. The average $H_{t}$ is $7.1 \times 10^{3} \mathrm{~A} / \mathrm{m}$ with a standard deviation of $484 \mathrm{~A} / \mathrm{m}$

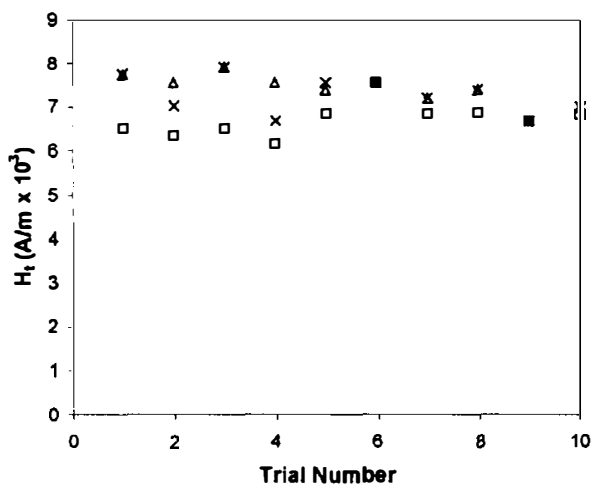

Figure 5. Threshold $H_{\text {ext }}$ for the Type I structures. The Type I structure has two stable positions. Tests were conducted on 3 identical Type I structures for 10 trials each.

The $H_{1}$ is found to be dependant on an initial angular displacement. Some flaps have an initial angle, $\theta \approx 8^{\circ}$; for these, the average $H_{\text {r }}$ is $3.8 \times 10^{3} \mathrm{~A} / \mathrm{m}$ with the standard deviation being 324 $\mathrm{A} / \mathrm{m}$ for 30 trials. We conjecture that such a behavior is related to the fluid. At $\theta=0$, it is assumed that there is no fluid between the flap and the substrate. As the flap rotates, the space underneath it must be replaced with fluid. If $\theta$ is originally greater than $0^{\circ}$, fluid can fill the same space with less effort. As a result, less energy and a lower $H_{1}$ is required when the flap is initially deflected.

Unlike Type I structures, Type II structures can achieve a continuous range of angular displacements due to the counteracting spring loading created by the cantilever beam. Figure 6 shows the actuation behavior of three Type II structures with different cantilever-beam geometries and spring constants. The offset slab will not contact the cantilever-beam loading spring until the flap has been deflected by nearly $35^{\circ}$, as defined by slacks caused by removed sacrificial material. After the contact has been made, flap displacement increases continuously as $H_{e x t}$ is increased. The behavior of the Type II structures appear to have three regimes after the offset slab and the cantilever beam have made contact. For small values of $\theta$, increases in $H_{\text {ext }}$ result in slow linear increment of $\theta$. This could be attributed to partial magnetization of the Permalloy. Near $\theta=50^{\circ}$, the rate of change in $\theta$ with respect to $H_{e x t}$ begins to increase, indicating the Permalloy has become fully magnetized. The curve then begins to saturate again as the flap approaches $90^{\circ}$. This behavior can be explained by Eq. 1, which predicts that the magnitude of the magnetic torque will decrease with increased $\theta$. The general trends are the same for all other cantilever beam dimensions we have tested. As predicted by linear beam theory, flaps with stiffer cantilever beams require a stronger $H_{\text {ext }}$ to achieve $90^{\circ}$ displacements.

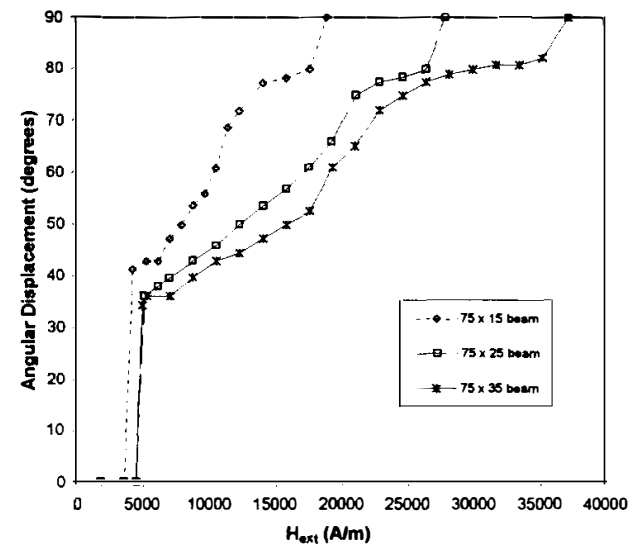

Figure 6. Behavior of the Type II structures. Type II structures are able to acheive a range of stable angular displacements. The data is for cantilever beams of length 75 ton and widths of 15,25, and 35 rom.

Type II structures with no added locking mechanisms can remain stable at $90^{\circ}$ after $H_{\text {ext }}$ has been removed. When the flap is at $90^{\circ}$, a flat portion of the offset slab is in contact with the cantilever beam. The applied restoring force provided by the cantilever beam to the slab is large enough to maintain the flap at $90^{\circ}$. We conjecture that the intrinsic stability of such an assembly is low as environmental factors (e.g. vibrations, fluid forces) can cause the flap to collapse.

Combinations of two or three Type I flaps were used to construct stable 3-D assemblies. An assembled device which uses two structures is shown in Figure 7 . This device consists of a primary flap and a secondary flap. The secondary flap contains a slot used to lock the primary flap and maintain a stable position utilizing frictional forces between flaps. These two flaps are lifted in a predetermined order (Fig. 8). Under a uniform magnetic field, the displacement of flaps is controlled by using different volumes of the magnetic pieces. The volume of Permalloy is $200 \times 40 \times 2.1$ $\mu \mathrm{m}^{3}$ on the primary flap and $100 \times 40 \times 2.1 \mu \mathrm{m}^{3}$ on the secondary flap. Both flaps are initially in plane (Fig. 8a) when there is no external magnetic field. As $H_{\text {ext }}$ is gradually increased, the primary flap will rise to $90^{\circ}$ (Fig. 8b) first. A further increase of $H_{\text {ext }}$ causes the secondary flap to rise to $90^{\circ}$ and lock the primary flap into a fixed position (Fig. 8c). 


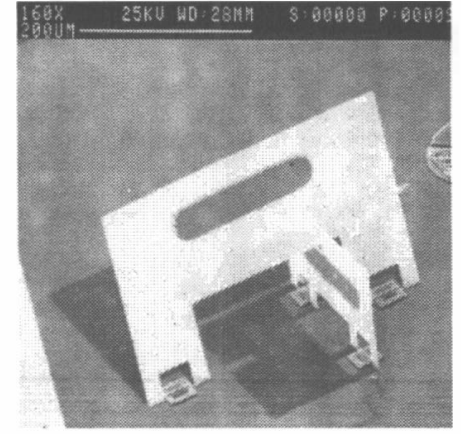

Figure 7. SEM micrograph of a 3-D device using two Type I structures. The sequence of actuation was controlled by the use of different Permalloy volumes.

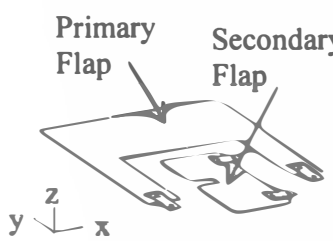

(a)
Frictional

Effects

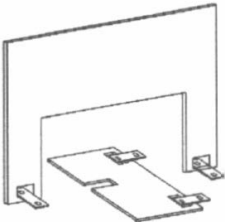

(b)

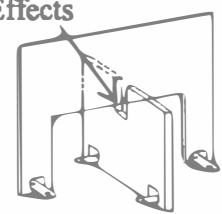

(c)
Figure 8. Schematic of the assembly process for the flap 3-D devices. (a) Both flaps in the resting position; (b) primary flap raised to $90^{\circ}$ at $H_{\text {ext }}=H_{1}$; (c) full 3-D assembly is achieved at $H_{\text {ext }}$ $=\mathrm{H}_{2}\left(\mathrm{H}_{2}>\mathrm{H}_{\downarrow}\right)$.

3-D assemblies utilizing three flaps, one primary and two secondary, have also been designed (Fig. 9). The primary flap has a tapered area and the secondary flaps contain locking slots. The advantage of this design is that the order of actuation is not critical to the assembly of the device. The device will assemble if all three flaps rise to $90^{\circ}$, regardless of the order of actuation. If the primary hinge rises to $90^{\circ}$ first, then the secondary hinges rise $90^{\circ}$ to lock the primary hinge into place. On the other hand, secondary hinges may rise to $90^{\circ}$ first. As the primary hinge rises to $90^{\circ}$, it pushes the secondary hinges outward and then locks into place when it reaches the slots. Both the two and three hinge assemblies are capable of remaining assembled after $H_{e x}$ is removed.

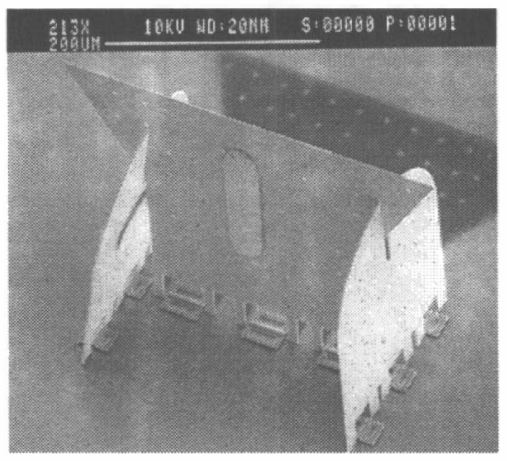

Figure 9. An SEM micrograph of a 3-D device using three Type I flaps. The sequence of actuation is not critical to the assembly of this device.

A primary advantage that magnetostatic actuation offers is the ability to control an array of devices in parallel with a uniform magnetic field. Figures 10 and 11 show examples of hinged microstructures that have been actuated with a linear sweep of $H_{\text {ext }}$

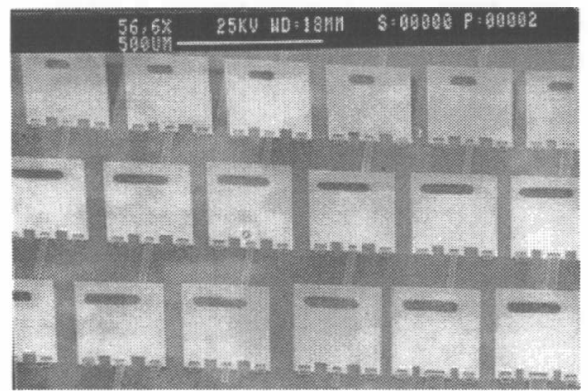

Figure 10. SEM micrograph of an array of Type II structures. The flaps are rotated $90^{\circ}$ and there is no $H_{\text {ext }}$ applied.

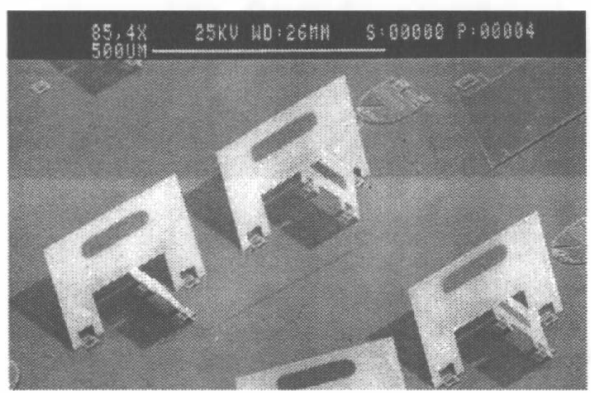

Figure 11. SEM micrograph of an array of 3-D devices. $H_{\text {ext }}$ has been removed.

\section{CONCLUSIONS}

Sequential and parallel actuation of hinged microstructures has been demonstrated through the use of magnetic actuation. The angular displacement of the structures can be controlled by varying the volume of magnetic material or the stiffness of a cantileverbeam loading device. The behavior of two types of hinged microstructures, using these principles, have been characterized. It was observed that Type I structures have two stable positions, while the inclusion of a cantilever spring in Type II structures allow them to achieve a range of stable angular displacements. The ability to control the angular displacement of individual hinged flaps under a global magnetic field has enabled us to assemble three dimensional devices.

\section{REFERENCES}

1. K. S. J Pister, M. W. Judy, S.R. Burgett, and R. S. Fearing. "Microfabricated hinges," Sensors and Actuators A, 33 (1992).

2. J.H. Comtois and V.M. Bright. "Surface micromachined polysilicon thermal actuator arrays and applications," Proceedings, IEEE Solid-State Sensors\&Actuators Workship, Hilton Head, SC, p. 174, 1996.

3. L. Fan, M.C. Wu, et al.. "Self-assembled microactuated XYZ stages for optical scanning and alignment," Transducer '97, Vol. 1, p. 319, 1997.

4. J. Shigley and C. Mischke, Mechanical Engineering Design, McGraw-Hill. NewYork, 1989.

5. C. Liu, T. Tsao, and Y. Tai. "Out-of-Plane Permalloy Magnetic Actuators for Delta-Wing Control.” MEMS '95, p. 7,1995.

6. C. Liu, T. Tsao, Y.C. Tai. "A High Yield Drying/Release Process for Surface Micromachined Polysilicon Structures," Transducers '97, June 1997. 